\title{
New Representations of Spherical Indicatricies of Bertrand Curves in Minkowski 3-Space
}

\author{
İsmail Aydemir and Furat Yerlikaya \\ Department of Mathematics, Art and Science Faculty, Ondokuz Mayis University, Kurupelit campus, 55190 Samsun, Turkey \\ Correspondence should be addressed to Firat Yerlikaya; firat_ykaya@hotmail.com
}

Received 26 September 2014; Accepted 24 December 2014

Academic Editor: Isaac Pesenson

Copyright (C) 2015 İ. Aydemir and F. Yerlikaya. This is an open access article distributed under the Creative Commons Attribution License, which permits unrestricted use, distribution, and reproduction in any medium, provided the original work is properly cited.

\begin{abstract}
We obtained a new representation for timelike Bertrand curves and their Bertrand mate in 3-dimensional Minkowski space. By using this representation, we expressed new representations of spherical indicatricies of Bertrand curves and computed their curvatures and torsions. Furthermore in case the indicatricies of a Bertrand curve are slant helices, we investigated some new characteristic features of these curves.
\end{abstract}

\section{Introduction}

In the early 1900s, Bertrand had worked on special curves, which were then referred to by his name. He examined these curves and their applications to both differential equations in analytical mechanics and thermodynamics [1]. Bertrand curve is defined as a particular curve, which shares its principal normal vector with another special curve that called Bertrand pair. Also, there is a linear relation between the curvature and the torsion. In three-dimensional euclidean space, a helix is characterized as a curve whose tangent line makes a constant angle with a fixed direction. Lancret expressed the result, which describes helices, a curve is a helix if and only if the ratio $\tau / \kappa$ is constant in 1802, and Saint Venant proved it in 1845. There are many highly interesting applications of helices such as helical structures in fractal geometry, DNA spirals, collagen triple helix and the helical ladder structures in architecture [2-5]. Izumiya and Takeuchi defined Slant helices. A Slant helix is a curve whose principal normal vector makes a constant angle with a fixed direction [6].

In three-dimensional Minkowski space, curves are classified into three groups: spacelike, timelike, and lightlike. In this paper, we examined timelike Bertrand curves and their timelike Bertrand mate with a geometric point of view. We obtained a new representation for timelike Bertrand curves and their Bertrand mate in 3-dimensional Minkowski space.
By using this representation, we expressed new representations of spherical indicatricies of Bertrand curves and computed their curvatures and torsions. Furthermore, in case the indicatricies of a Bertrand curve are slant helices, we investigated some new characteristic features of these curves.

3-dimensional Minkowski space $\mathbb{R}_{1}^{3}$ is the $\mathbb{R}^{3}$ vector space endowed with Lorentzian inner product given by

$$
\langle x, y\rangle=-x_{1} y_{1}+x_{2} y_{2}+x_{3} y_{3},
$$

where $x=\left(x_{1}, x_{2}, x_{3}\right)$ and $y=\left(y_{1}, y_{2}, y_{3}\right) \in \mathbb{R}^{3}$.

A vector is called a spacelike vector if $\langle x, x\rangle>0$, a timelike vector if $\langle x, x\rangle<0$, and a lightlike vector if $x \neq 0$ and $\langle x, x\rangle=0$. Likewise, an arbitrary curve $\alpha(t)$ in $\mathbb{R}_{1}^{3}$ is addressed as spacelike, timelike, or null, if its velocity vectors $\alpha^{\prime}(t)$ are respectively spacelike, timelike, or null, for every $t \in I \subset$ $\mathbb{R}$. For a vector $x \in \mathbb{R}_{1}^{3}$, the norm of $x$ is defined by

$$
\|x\|=\sqrt{|\langle x, x\rangle|} .
$$

In addition, $x$ is called a unit vector if its norm is equal to 1 . The Lorentzian sphere and hyperbolic sphere of radius 1 in 3-dimensional Minkowski space are, respectively, given by

$$
\begin{aligned}
S_{1}^{2} & =\left\{x=\left(x_{1}, x_{2}, x_{3}\right) \in \mathbb{R}_{1}^{3}:\langle x, x\rangle=1\right\}, \\
H_{1}^{2} & =\left\{x=\left(x_{1}, x_{2}, x_{3}\right) \in \mathbb{R}_{1}^{3}:\langle x, x\rangle=-1\right\} .
\end{aligned}
$$


For any $x=\left(x_{1}, x_{2}, x_{3}\right)$ and $y=\left(y_{1}, y_{2}, y_{3}\right) \in \mathbb{R}_{1}^{3}$, the vectorial product of $x$ and $y$ is defined by

$$
x \times y=\left(x_{3} y_{2}-x_{2} y_{3}, x_{3} y_{1}-x_{1} y_{3}, x_{1} y_{2}-x_{2} y_{1}\right) .
$$

We denote the moving Frenet frame along the curve $\alpha$ by $\{T(t), N(t), B(t)\}$ where $T, N$, and $B$ are the tangent, the principal normal and the binormal vector of the curve $\alpha$, respectively. Let $\alpha$ be a unit speed timelike space curve with curvature $\kappa$, torsion $\tau$ and Frenet vector fields of $\alpha$ be $\{T, N, B\}$ where $T$ is timelike and $N, B$ are spacelike vector fields. Then, Frenet formulas are

$$
\left[\begin{array}{c}
T^{\prime} \\
N^{\prime} \\
B^{\prime}
\end{array}\right]=\left[\begin{array}{ccc}
0 & \kappa & 0 \\
\kappa & 0 & \tau \\
0 & -\tau & 0
\end{array}\right]\left[\begin{array}{c}
T \\
N \\
B
\end{array}\right] .
$$

For given any two vectors $x, y \in \mathbb{R}_{1}^{3}$, if $x$ and $y$ are positive (negative) timelike vectors then there is a unique nonnegative real number $\theta$ such that

$$
\langle x, y\rangle=\|x\|\|y\| \cosh \theta .
$$

Moreover, this unique nonnegative real number $\theta$ is called The Lorentzian timelike angle between $x$ and $y$. If $x$ is a spacelike vector and $y$ be a positive timelike vector then there is a unique nonnegative real number $\theta$ such that

$$
|\langle x, y\rangle|=\|x\|\|y\| \sinh \theta .
$$

Therefore, this unique nonnegative real number $\theta$ is called The Lorentzian timelike angle between $x$ and $y$ (see [7]).

A unit speed curve $\alpha$ is called a slant helix if there exists a constant vector field $U$ in $\mathbb{R}_{1}^{3}$ such that $\langle N(s), U\rangle$ is constant [8].

Theorem 1. Let $\alpha$ be unit speed timelike curve in $\mathbb{R}_{1}^{3}$. Then, $\alpha$ is a slant helix if and only if either one of the next two functions

$$
\frac{\kappa^{2}}{\left(\tau^{2}-\kappa^{2}\right)^{3 / 2}}\left(\frac{\tau}{\kappa}\right)^{\prime}
$$

or

$$
\frac{\kappa^{2}}{\left(\kappa^{2}-\tau^{2}\right)^{3 / 2}}\left(\frac{\tau}{\kappa}\right)^{\prime}
$$

is constant everywhere $\tau^{2}-\kappa^{2}$ does not vanish [8].

\section{New Representations of Timelike Bertrand Curves}

Let $\alpha$ be a unit speed timelike curve. If there exists a timelike curve $\alpha^{*}$ whose principal normal vector coincides with that of $\alpha$, then $\alpha$ is called a timelike Bertrand curve. The pair $\left(\alpha, \alpha^{*}\right)$ is said to be a timelike Bertrand pair. Let $\alpha$ be a timelike Bertrand curve and let $\alpha^{*}$ be a timelike Bertrand mate of $\alpha$ and we denote the moving Frenet frame along the curve $\alpha$ by $\{T, N, B\}$ and along the curve $\alpha^{*}$ by $\left\{T^{*}, N^{*}, B^{*}\right\}$. Thus, the tangent vector fields and the binormal vector fields are, respectively, related by

$$
\begin{gathered}
T=\cosh \theta T^{*}-\sinh \theta B^{*}, \\
B=-\sinh \theta T^{*}+\cosh \theta B^{*}
\end{gathered}
$$

for some functions $\theta=\theta(s)$. Furthermore, $\theta$ is the angle between the tangent vectors $T$ and $T^{*}$. Similar relations can be given as follows for the curvatures and torsions:

$$
\begin{aligned}
& \kappa=\cosh \theta \frac{d s}{d s^{*}} \kappa^{*}+\sinh \theta \frac{d s}{d s^{*}} \tau^{*}, \\
& \tau=\sinh \theta \frac{d s}{d s^{*}} \kappa^{*}+\cosh \theta \frac{d s}{d s^{*}} \tau^{*},
\end{aligned}
$$

where $\kappa, \kappa^{*}$ and $\tau, \tau^{*}$ are the curvatures and torsions of $\alpha$ and $\alpha^{*}$, respectively.

Furthermore, let $\left(\alpha, \alpha^{*}\right)$ be a nonhelical timelike Bertrand pair; $s$ and $s^{*}$ are arclength parameters of $\alpha$ and $\alpha^{*}$, respectively. Hence, $\alpha(s)=\alpha^{*}\left(s^{*}\right)-\lambda \varepsilon N^{*}$ and $\alpha^{*}\left(s^{*}\right)=\alpha(s)+\lambda N$. Let $\alpha$ be a timelike Bertrand curve with a timelike Bertrand mate curve $\alpha^{*}$; then,

$$
\lambda=\frac{\varepsilon g^{*}}{\kappa^{*}\left(g^{*}-f^{*}\right)},
$$

where

$$
f^{*}=\frac{\tau^{*}}{\mathcal{K}^{*}}, \quad g^{*}=\frac{\tau^{* \prime}}{\kappa^{* \prime}}
$$

and $\varepsilon= \pm 1$.

Moreover, we can give the relation between the arclength parameters $s$ and $s^{*}$ of $\alpha$ and $\alpha^{*}$, respectively, as follows:

$$
s=\int \frac{f^{*} \sqrt{1-g^{* 2}}}{g^{*}-f^{*}} d s^{*} .
$$

Theorem 2. Let $\alpha$ be a timelike Bertrand curve and let $\alpha^{*}$ be a nonhelical timelike Bertrand mate of $\alpha$. We denote the moving Frenet frame along the curve $\alpha$ by $\{T, N, B\}$ and along the curve $\alpha^{*}$ by $\left\{T^{*}, N^{*}, B^{*}\right\}$. Then,

$$
\begin{aligned}
& T=\frac{-1}{\sqrt{1-g^{* 2}}}\left\{T^{*}+g^{*} B^{*}\right\}, \\
& N=\varepsilon N^{*}, \\
& B=\frac{-\varepsilon}{\sqrt{1-g^{* 2}}}\left\{g^{*} T^{*}+B^{*}\right\}, \\
& \kappa=\frac{-\varepsilon\left(1-g^{*} f^{*}\right)\left(g^{*}-f^{*}\right)}{f^{*}\left(1-g^{* 2}\right)}, \quad \tau=\frac{\kappa^{*}\left(g^{*}-f^{*}\right)^{2}}{f^{*}\left(1-g^{* 2}\right)},
\end{aligned}
$$

where $\varepsilon \pm 1,\left|\kappa^{* \prime}\right|>\left|\tau^{* \prime}\right|$, and $\kappa, \kappa^{*}$ and $\tau, \tau^{*}$ are the curvatures and torsions of $\alpha$ and $\alpha^{*}$, respectively. 
The geodesic curvature of the principal image of the principal normal indicatrix of $\alpha$ is

$$
\sigma=\frac{\varepsilon \kappa^{* \prime}\left(g^{*}-f^{*}\right)}{\kappa^{* 2}\left(1-f^{* 2}\right)^{3 / 2}} .
$$

Also, if we compute the derivative $\left(\tau^{*} / \kappa^{*}\right)^{\prime}$ and put it in equation $\sigma^{*}=\left(\kappa^{* 2} /\left(\kappa^{* 2}-\tau^{* 2}\right)^{3 / 2}\right)\left(\tau^{*} / \kappa^{*}\right)^{\prime}$, we obtain

$$
\sigma^{*}=\frac{\kappa^{* \prime}\left(g^{*}-f^{*}\right)}{\kappa^{* 2}\left(1-f^{* 2}\right)^{3 / 2}} \text {. }
$$

So we have $\sigma^{*}=\sigma / \varepsilon$.

Now, we can give the following result.

Corollary 3. Let $\alpha$ be a timelike Bertrand curve and $\alpha^{*}$ be a nonhelical timelike Bertrand mate of $\alpha$. Bertrand curve $\alpha$ is a slant helix if and only if its mate curve $\alpha^{*}$ is a slant helix.

Theorem 4. Let $\alpha$ be a timelike Bertrand curve and $\alpha^{*}$ be a nonhelical timelike Bertrand mate of $\alpha$ and $\left|\kappa^{* \prime}\right|>\left|\tau^{* \prime}\right|$. Then, $g^{*}$ is a constant.

Proof. If we differentiate the equation $\alpha(s)=\alpha^{*}\left(s^{*}\right)-\lambda \varepsilon N^{*}$ with respect to $s^{*}$, we obtain

$$
\alpha^{\prime}(s)=\frac{d s^{*}}{d s}\left(1-\lambda \varepsilon \kappa^{*}\right) T^{*}-\frac{d s^{*}}{d s} \lambda \varepsilon \tau^{*} B^{*} .
$$

By substituting $\lambda=\varepsilon g^{*} / \kappa^{*}\left(g^{*}-f^{*}\right)$ and $d s / d s^{*}=$ $f^{*} \sqrt{1-g^{* 2}} /\left(g^{*}-f^{*}\right)$ in the equation and choosing

$$
p_{1}=\frac{1}{\sqrt{1-g^{* 2}}}, \quad p_{2}=\frac{g^{*}}{\sqrt{1-g^{* 2}}},
$$

we can write

$$
\alpha^{\prime}(s)=-\frac{1}{\sqrt{1-g^{* 2}}} T^{*}+\frac{g^{*}}{\sqrt{1-g^{* 2}}} B^{*}=-p_{1} T^{*}+p_{2} B^{*} ;
$$

then, if we differentiate the last equation again with respect to $s^{*}$, we get

$$
\alpha^{\prime \prime}(s)=\frac{d \alpha^{\prime}(s)}{d s^{*}}=-p_{1}^{\prime} T^{*}-\left\{p_{1} \kappa^{*}+p_{2} \tau^{*}\right\} N^{*}+p_{2}^{\prime} B^{*} .
$$

Since $\alpha^{\prime \prime}, T^{*}$, and $B^{*}$ are linear independent, $p_{1}^{\prime}=0$ and $p_{2}^{\prime}=0$. Thus, $p_{1}$ and $p_{2}$ are constants. Therefore, $g^{*}$ is a constant.

\section{New Representations of Spherical Indicatrix of Timelike Bertrand Curves}

Definition 5. Let $\alpha$ be a unit speed regular curve and let $T, N$, and $B$ be Frenet vectors in Minkowski 3-space. Thus, there exist unit tangent vectors along the curve $\alpha$. These unit tangent vectors generate a curve $\alpha_{t}=T$ on the unit Lorentzian sphere (or hyperbolic sphere). The curve $\alpha_{t}$ is called the Tangent indicatrix of the curve $\alpha$. Similarly, we can define the principal normal and binormal indicatrix of the curve $\alpha$.

3.1. The Tangent Indicatrix of Timelike Bertrand Curves. If we take a nonhelical timelike Bertnard pair $\left(\alpha, \alpha^{*}\right)$ in the above definition, we get the tangent indicatrix of a timelike Bertrand curve such that

$$
\alpha_{t}=\frac{-1}{\sqrt{1-g^{* 2}}}\left\{T^{*}+g^{*} B^{*}\right\} .
$$

Theorem 6. Let $\alpha$ be a timelike Bertrand curve and $\left|\tau^{*}\right|>$ $\kappa^{*}$. If the Frenet frame of the tangent indicatrix $\alpha_{t}=T$ of the timelike Bertrand curve is $\left\{T_{t}, N_{t}, B_{t}\right\}$ then

$$
\begin{aligned}
& T_{t}=-N^{*}, \quad N_{t}=\frac{-1}{\sqrt{f^{* 2}-1}}\left\{T^{*}+f^{*} B^{*}\right\}, \\
& B_{t}=\frac{-1}{\sqrt{f^{* 2}-1}}\left\{f^{*} T^{*}+B^{*}\right\} .
\end{aligned}
$$

From (22), we know $\alpha_{t}=T=\left(-1 / \sqrt{1-g^{* 2}}\right)\left\{T^{*}+\right.$ $\left.g^{*} B^{*}\right\}$. If we differentiate this equation with respect to $s^{*}$ and reorganize, we have

$$
s_{t}=\int \frac{\kappa^{*}\left(1-f^{*} g^{*}\right)}{\sqrt{1-g^{* 2}}} d s^{*},
$$

where $\left|\tau^{*}\right|>\kappa^{*}$ and $\left|\kappa^{* \prime}\right|>\left|\tau^{* \prime}\right|$. With a similar way, we can express following relation between the arclength parameters $s_{t}$ and $s$ of $\alpha_{t}$ and $\alpha$, respectively:

$$
s_{t}=\int \frac{\kappa^{*}\left(1-f^{*} g^{*}\right)\left(g^{*}-f^{*}\right)}{f^{*}\left(1-g^{* 2}\right)} d s .
$$

Theorem 7. Let $\alpha$ be a timelike Bertrand curve and let $\alpha^{*}$ be a nonhelical timelike Bertrand mate of $\alpha,\left|\tau^{*}\right|>\kappa^{*}$ and $\left|\kappa^{* \prime}\right|>$ $\left|\tau^{* \prime}\right|$. Thus, curvature and torsion of the tangent indicatrix $\alpha_{t}$ of the timelike Bertrand curve are

$$
\begin{gathered}
\kappa_{t}=\frac{\sqrt{f^{* 2}-1} \sqrt{1-g^{* 2}}}{\left(1-f^{*} g^{*}\right)}, \\
\tau_{t}=\frac{-\kappa^{* \prime} \sqrt{1-g^{* 2}}\left(g^{*}-f^{*}\right)}{\kappa^{* 2}\left(1-f^{*} g^{*}\right)\left(f^{* 2}-1\right)},
\end{gathered}
$$

respectively.

Corollary 8. Let $\left(\alpha, \alpha^{*}\right)$ be a nonplanar and nonhelical timelike Bertrand pair in $\mathbb{R}_{1}^{3}$. Thus, Bertrand curve is a slant helix if and only if the tangent indicatrix of the Bertrand curve is a helix. 
Theorem 9. Let $\alpha$ be a timelike Bertrand curve and let $\alpha^{*}$ be a nonhelical timelike Bertrand mate of $\alpha$. Then, the geodesic curvature of the principal image of the principal normal indicatrix of $\alpha_{t}$ is

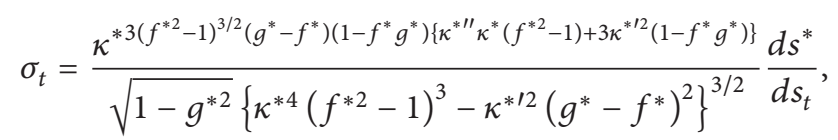

where $\left|\kappa^{* \prime}\right|>\left|\tau^{* \prime}\right|$ and $\left|\tau^{*}\right|>\kappa^{*}$.

Corollary 10. Let $\alpha$ be a timelike Bertrand curve and let $\alpha^{*}$ be a nonhelical timelike Bertrand mate of $\alpha$. Then, the tangent indicatrix of the timelike Bertrand curve is a helix if and only if

$$
\kappa^{* \prime \prime} \kappa^{*}\left(f^{* 2}-1\right)+3 \kappa^{* 2}\left(1+f^{*} g^{*}\right)=0
$$

is satisfied.

3.2. The Principal Normal Indicatrix of Timelike Bertrand Curves. It is known that the principal normal indicatrix of a timelike Bertrand curve is

$$
\alpha_{n}=\varepsilon N^{*} \text {. }
$$

Theorem 11. Let $\alpha$ be a timelike Bertrand curve and $\left|\tau^{*}\right|>$ $\kappa^{*}$. If the Frenet frame of the principal normal indicatrix of the timelike Bertrand curve is $\left\{T_{n}, N_{n}, B_{n}\right\}$ then

$$
\begin{aligned}
& T_{n}=\frac{\varepsilon}{\sqrt{f^{* 2}-1}}\left\{T^{*}+f^{*} B^{*}\right\} \\
& \begin{aligned}
N_{n}= & \frac{-\varepsilon}{\rho \sqrt{f^{* 2}-1}}\left\{f^{*} \kappa^{* \prime}\left(g^{*}-f^{*}\right) T^{*}\right. \\
& +\kappa^{* 2}\left(f^{* 2}-1\right)^{2} N^{*} \\
& \left.+\kappa^{* \prime}\left(g^{*}-f^{*}\right) B^{*}\right\}, \\
B_{n}=\frac{-1}{\rho}\left\{-f^{*} \kappa^{* 2}\left(f^{* 2}-1\right) T^{*}+\kappa^{* \prime}\left(g^{*}-f^{*}\right) N^{*}\right. & \left.+\kappa^{* 2}\left(f^{* 2}-1\right) B^{*}\right\},
\end{aligned}
\end{aligned}
$$

where $\rho=\sqrt{\left|\kappa^{* 4}\left(f^{* 2}-1\right)^{3}-\kappa^{* \prime 2}\left(g^{*}-f^{*}\right)^{2}\right|}$.

If $\alpha$ is a timelike Bertrand curve and $\alpha^{*}$ is a nonhelical timelike Bertrand mate of $\alpha,\left|\tau^{*}\right|>\kappa^{*}$ and $\left|\kappa^{* \prime}\right|>\left|\tau^{* \prime}\right|$, then

$$
\begin{aligned}
& s_{n}=\int \kappa^{*} \sqrt{f^{* 2}-1} d s^{*}, \\
& s_{n}=\int \frac{\kappa^{*} \sqrt{f^{* 2}-1}\left(g^{*}-f^{*}\right)}{f^{*} \sqrt{1-g^{* 2}}} d s .
\end{aligned}
$$

Furthermore, the curvature and the torsion of the principal normal indicatrix $\alpha_{n}=N$ of the timelike Bertrand curve are

$$
\begin{aligned}
\kappa_{n}= & \frac{\rho}{\kappa^{* 2}\left(f^{* 2}-1\right)^{3 / 2}}, \\
\tau_{n}= & \frac{\varepsilon\left(g^{*}-f^{*}\right)}{\rho^{2}} \\
& \cdot\left\{\left(3 \kappa^{*^{\prime} 2}-\kappa^{*} \kappa^{*^{\prime \prime}}\right)\left(1-f^{* 2}\right)-3 f^{*} \kappa^{*^{\prime} 2}\left(g^{*}-f^{*}\right)\right\},
\end{aligned}
$$

respectively, where $\rho=\sqrt{\left|\kappa^{* 4}\left(f^{* 2}-1\right)^{3}-\kappa^{* / 2}\left(g^{*}-f^{*}\right)^{2}\right|}$.

Theorem 12. Let $\alpha$ be a timelike Bertrand curve and let $\alpha^{*}$ be a nonhelical timelike Bertrand mate of $\alpha$. Then, the principal normal indicatrix of the timelike Bertrand curve is a helix if and only if

$$
\kappa^{* \prime \prime} \kappa^{*}\left(f^{* 2}-1\right)+3 \kappa^{* 2}\left(1-g^{*} f^{*}\right)=0
$$

is satisfied.

3.3. The Binormal Indicatrix of Timelike Bertrand Curves. It is known that the binormal indicatrix of a timelike Bertrand curve is

$$
\alpha_{b}=\frac{-\varepsilon}{\sqrt{1-g^{* 2}}}\left\{g^{*} T^{*}+B^{*}\right\}
$$

Theorem 13. Let $\alpha$ be a timelike Bertrand curve and $\left|\tau^{*}\right|>\kappa^{*}$. If the Frenet frame of the binormal indicatrix of the timelike Bertrand curve is $\left\{T_{b}, N_{b}, B_{b}\right\}$ then

$$
\begin{aligned}
& T_{b}=-N^{*}, \quad N_{b}=\frac{-\varepsilon}{\sqrt{f^{* 2}-1}}\left\{T^{*}+f^{*} B^{*}\right\}, \\
& B_{b}=\frac{\varepsilon}{\sqrt{f^{* 2}-1}}\left\{f^{*} T^{*}-B^{*}\right\} .
\end{aligned}
$$

Theorem 14. Let $\alpha$ be a timelike Bertrand curve and let $\alpha^{*}$ be a nonhelical timelike Bertrand mate of $\alpha,\left|\tau^{*}\right|>\kappa^{*}$, and $\left|\kappa^{* \prime}\right|>\left|\tau^{* \prime}\right|$. Then,

$$
s_{b}=\int \frac{\varepsilon \kappa^{*}\left(g^{*}-f^{*}\right)}{\sqrt{1-g^{* 2}}} d s^{*}, \quad s_{b}=\int \frac{\varepsilon \kappa^{*}\left(g^{*}-f^{*}\right)^{2}}{f^{*}\left(1-g^{* 2}\right)} d s .
$$

And the curvature and the torsion of the binormal indicatrix $\alpha_{b}$ of the timelike Bertrand curve are

$$
\kappa_{b}=\frac{\sqrt{1-g^{* 2}} \sqrt{f^{* 2}-1}}{\left(g^{*}-f^{*}\right)}, \quad \tau_{b}=\frac{\kappa^{* \prime} \sqrt{1-g^{* 2}}}{\kappa^{* 2}\left(f^{* 2}-1\right)}
$$

respectively. 
As a result of this theorem, we have the following.

Corollary 15. A Bertrand curve is a slant helix if and only if the binormal indicatrix of the Bertrand curve is a helix.

Theorem 16. Let $\alpha$ be a timelike Bertrand curve and let $\alpha^{*}$ be a nonhelical timelike Bertrand mate of $\alpha$. The geodesic curvature of the principal image of the principal normal indicatrix of $\alpha_{b}$ is

$$
\begin{aligned}
& \sigma_{b} \\
& =\left(\left(\kappa^{* 3}\left(f^{* 2}-1\right)^{3 / 2}\left(g^{*}-f^{*}\right)^{2}\right.\right. \\
& \left.\cdot\left\{\kappa^{* \prime \prime} \kappa^{*}\left(f^{* 2}-1\right)+3 \kappa^{* / 2}\left(1-g^{*} f^{*}\right)\right\}\right) \\
& \cdot\left(\sqrt{1-g^{* 2}}\right) \\
& \left.\left.\cdot\left\{\kappa^{* 4}\left(f^{* 2}-1\right)^{3}-\kappa^{* / 2}\left(g^{*}-f^{*}\right)^{2}\right\}^{3 / 2}\right)^{-1}\right) \frac{d s^{*}}{d s_{b}},
\end{aligned}
$$

where $\left|\kappa^{* \prime}\right|>\left|\tau^{* \prime}\right|$ and $\left|\tau^{*}\right|>\kappa^{*}$.

Corollary 17. Let $\alpha$ be a timelike Bertrand curve and let $\alpha^{*}$ be a nonhelical timelike Bertrand mate of $\alpha$. Then, the binormal indicatrix of the timelike Bertrand curve is a helix if and only if

$$
\kappa^{* \prime \prime} \kappa^{*}\left(f^{* 2}-1\right)+3 \kappa^{* 2}\left(1-g^{*} f^{*}\right)=0
$$

is satisfied.

\section{Conflict of Interests}

The authors declare that there is no conflict of interests regarding the publication of this paper.

\section{References}

[1] J. Bertrand, "La thèories des courbes a double courbure," Journal de Mathématiques Pures et Appliquées, vol. 9, no. 15, pp. 332-350, 1850.

[2] N. Chouaieb, A. Goriely, and J. H. Maddocks, "Helices," Proceedings of the National Academy of Sciences of the United States of America, vol. 103, no. 25, pp. 9398-9403, 2006.

[3] C. D. Toledo-Suárez, "On the arithmetic of fractal dimension using hyperhelices," Chaos, Solitons and Fractals, vol. 39, no. 1, pp. 342-349, 2009.

[4] J. D. Watson and F. H. C. Crick, "Genetical implications of the structure of deoxyribonucleic acid," Nature, vol. 171, pp. 964967, 1953.

[5] A. A. Lucas and P. Lambin, "Diffraction by DNA, carbon nanotubes and other helical nanostructures," Reports on Progress in Physics, vol. 68, no. 5, pp. 1181-1249, 2005.

[6] S. Izumiya and N. Takeuchi, "New special curves and developable surfaces," Turkish Journal of Mathematics, vol. 28, no. 2, pp. 153-163, 2004.
[7] J. Walrave, Curves and surfaces in Minkowski space [Doctoral thesis], Katholieke Universiteit Leuven, Faculty of Science, Leuven, Belgium, 1995.

[8] A. T. Ali and R. Lopez, "On Slant Helices in Minkowski Space $\mathrm{E}_{1}^{3}$," Journal of the Korean Mathematical Society, vol. 48, no. 1, pp. 159-167, 2011. 


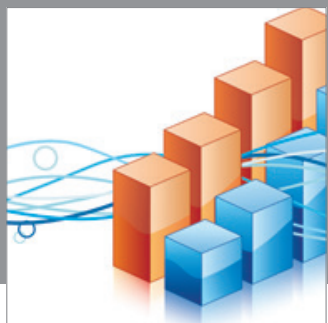

Advances in

Operations Research

mansans

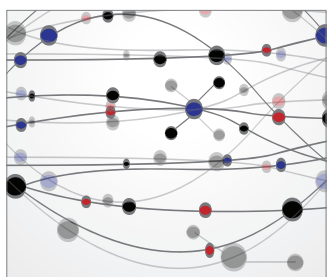

The Scientific World Journal
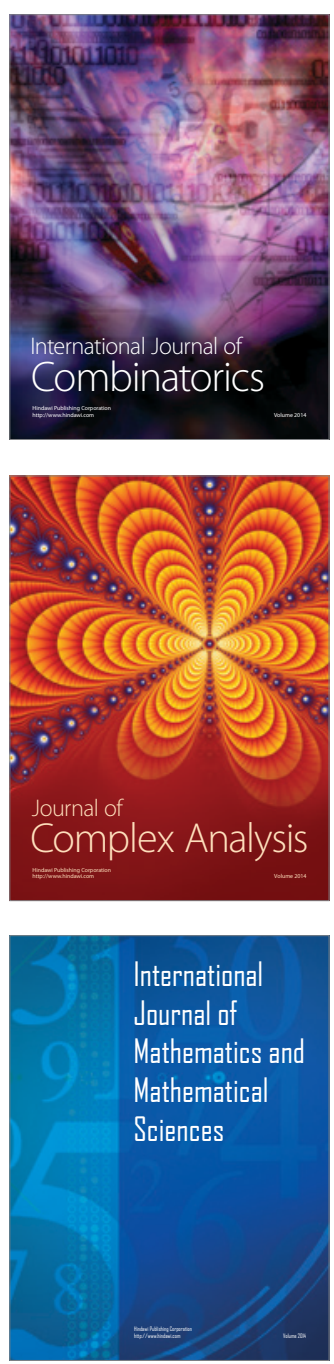
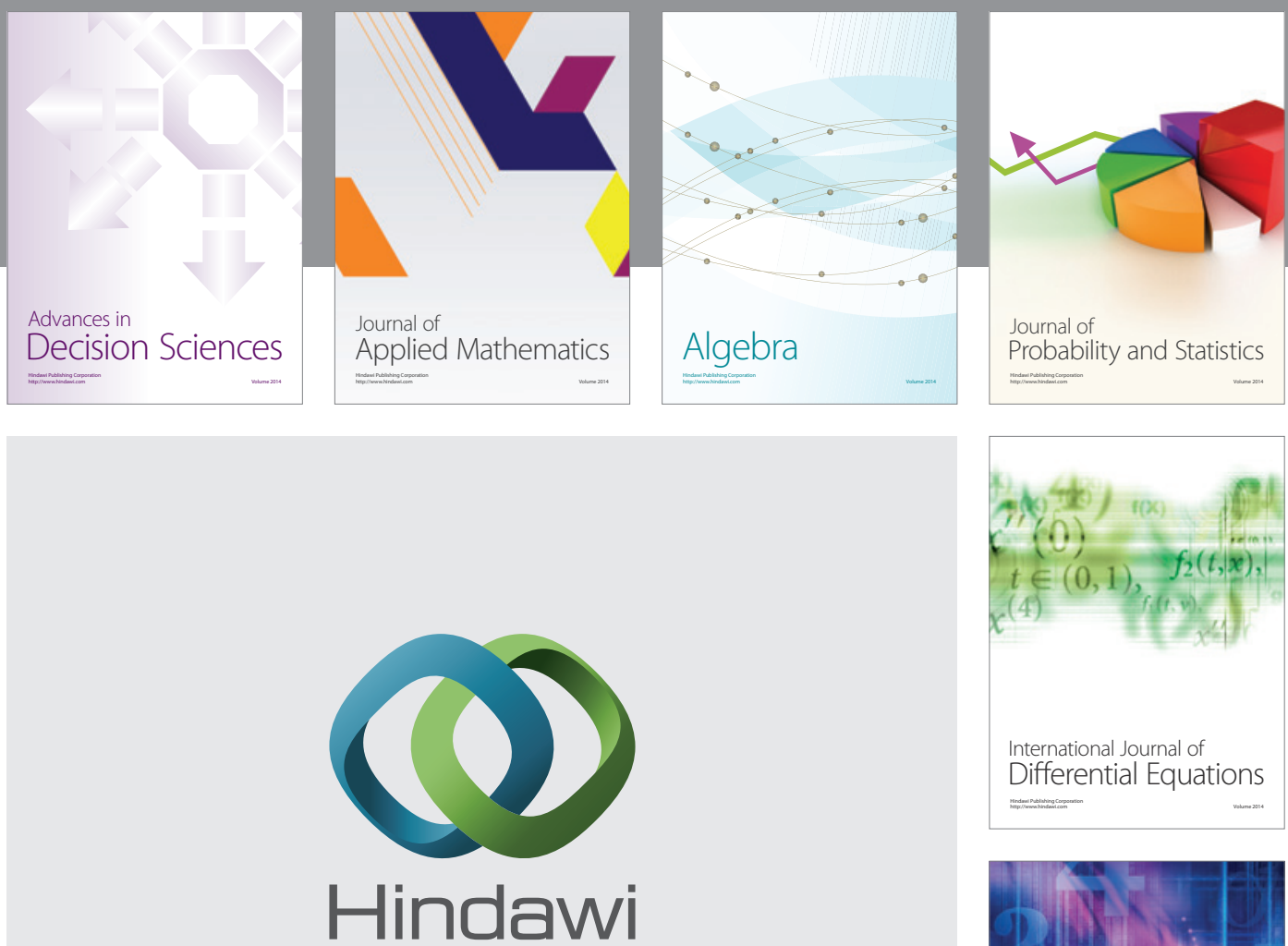

Submit your manuscripts at http://www.hindawi.com
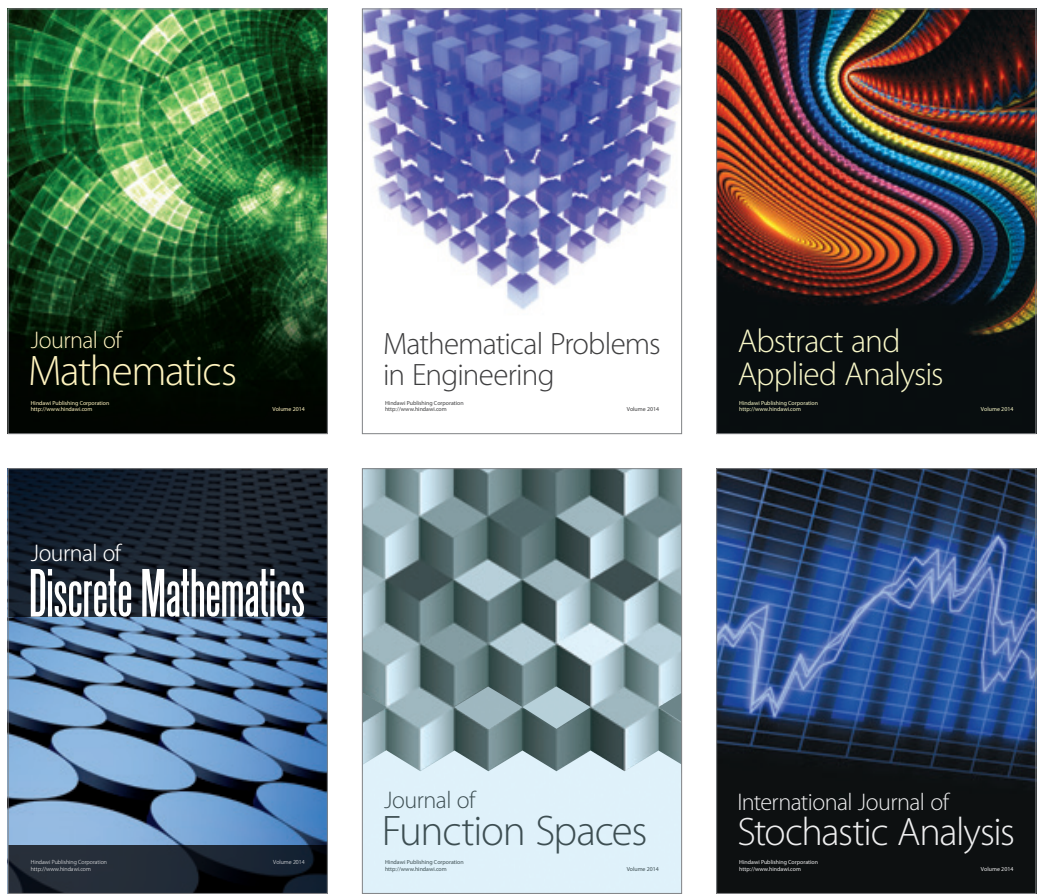

Journal of

Function Spaces

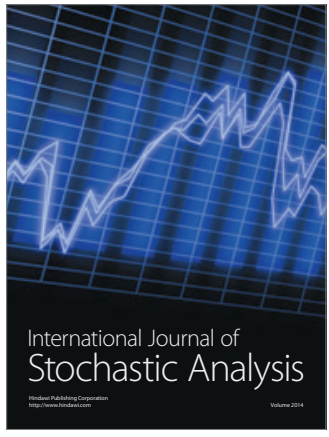

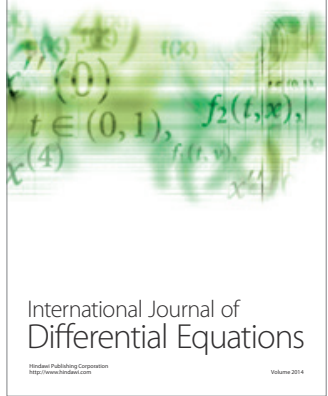
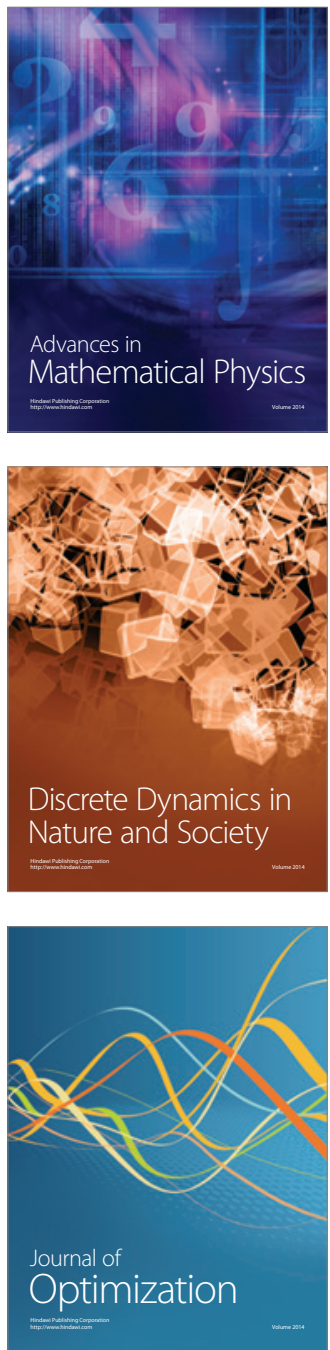